\title{
The association between the levels of CRP, IL-10, PLA2, Fbg and prognosis in traumatic fracture of lower limb
}

\author{
JING JIAO, JUN-WEN WANG, FEI XIAO and YU-CHENG HUANG \\ Department of Orthopedics, Wuhan Pu'ai Hospital, Wuhan, Hubei 430034, P.R. China
}

Received August 10, 2016; Accepted September 20, 2016

DOI: $10.3892 /$ etm.2016.3746

\begin{abstract}
The aim of the present study was to examine changes of sera levels of C-reactive protein (CRP), interleukin-10 (IL-10), phospholipase A2 (PLA2) and fibrinogen $\beta$ polypeptide chain gene (Fbg) in patients with traumatic fracture of lower limb, and to evaluate their association with prognosis. The changes in sera levels of CRP, IL-10, PLA2 and Fbg were observed at the time of injury, $24 \mathrm{~h}$, and 5 and 7 days after surgery in 90 patients with traumatic fracture of lower limb. In addition, 50 cases, who presented for health examination, were included as the normal controls. The expression of sera levels of CRP, IL-10, PLA2 and Fbg in patients with traumatic fracture of lower limb, was markedly higher than that in the normal controls prior to surgery $(\mathrm{P}<0.05)$. The concentration of CRP significantly increased within $24 \mathrm{~h}$ after emergency, but decreased gradually as the wound healed, compared to the controls. Pre- and postoperative IL-10 levels increased within $24 \mathrm{~h}$ and then decreased gradually. The level of PLA2 in patients before and after surgery was increased, and then decreased gradually. The level of Fbg in patients with trauma was increased after $24 \mathrm{~h}$ and then decreased, and increased gradually. The correlation of serum CRP and IL-10 levels $(r=0.634, P<0.05)$, and that of PLA2 and IL-10 levels $(r=0.617, P<0.05)$ were positive. In conclusion, the expression of CRP, IL-10, PLA2 and Fbg levels in traumatic fracture of lower limb markedly increased and was closely associated with prognosis. CRP, IL-10, PLA2 and Fbg levels may therefore serve as useful indexes in determining the progression and prognosis of patients with traumatic fracture of lower limb.
\end{abstract}

\section{Introduction}

Lower extremity trauma is a type of common orthopaedic trauma, and the key of its treatment is for the limb to be

Correspondence to: Dr Jing Jiao, Department of Orthopedics, Wuhan Pu'ai Hospital, 144 Hanzhong Road, Wuhan, Hubei 430034, P.R. China

E-mail: realzebra@qq.com

Key words: lower extremity trauma, C-reactive protein, interleukin-10, phospholipase A2, fibrinogen $\beta$ polypeptide chain gene, condition prediction rescued in time. Delayed treatment may lead to loss of limb function and permanent disability as a result of limb amputation (1-3). However, overemphasis on the rescue of injured lower extremity may result in ignorance of the general body condition, cause adverse outcomes, and induce severe complications that are unfavorable for prognosis (4). Therefore, scientific judgment of the severity and prognosis of lower extremity trauma should be given high attention, and it is imperative in the study of orthopaedic trauma.

Previous findings have shown that more serious lower extremity trauma may lead to an increased incidence of complication, infection, and organ dysfunction syndrome and be life threatening $(5,6)$. Indicators of C-reactive protein (CRP), interleukin-10 (IL-10), phospholipase A2 (PLA2) and fibrinogen $\beta$ polypeptide chain gene (Fbg) are critical inflammatory factors or important enzymes of inflammatory response. Investigations on how these indicators are used for predicting progression of lower extremity trauma have been previously conducted (7-9).

The present study focused on the predictive value of indicators mentioned above to determine the progression of lower extremity trauma and is expected to provide theoretical reference for the treatment of lower extremity trauma.

\section{Subjects and methods}

General data. In total, 90 cases of lower extremity trauma were admitted to the Wuhan Pu'ai Hospital (Hubei, China) from January, 2015 to December, 2015, and served as the study group. The study group comprised 58 men and 42 women, with an age range of 37-76 years, and an average age of $45.4 \pm 2.3$ years; ISS grading of 12-18, averaging, 14.6 \pm 1.5 ; $0.5-5 \mathrm{~h}$ of clinical treatment after injury, averaging, $3.14 \pm 0.6 \mathrm{~h}$; factors leading to trauma including traffic injuries (50 cases), falling injuries (34 cases), and others (6 cases); fracture types including femoral neck fractures (33 cases), intertrochanteric fractures (25 cases), femoral upper-part fractures (20 cases), and acetabular fractures (12 cases). The subjects had no diseases related to heart, kidney and other major organs, or orthopaedic surgery contraindications and history of mental disorder. Fifty subjects presenting for physical examination during the same period were selected as the normal control group. The normal control group comprised 26 men and 24 women, with an age range of 38-75 years and an average age of $46.1 \pm 2.6$ years. 
Table I. Comparison of CRP, IL-1, PLA2 and Fbg of the two groups.

\begin{tabular}{lcccc}
\hline Groups & CRP $(\mathrm{mg} / \mathrm{l})$ & $\mathrm{IL}-10(\mu \mathrm{g} / \mathrm{l})$ & PLA2 $(\mathrm{pg} / \mathrm{l})$ & $\mathrm{Fbg}(\mathrm{g} / \mathrm{l})$ \\
\hline Research & $43.32 \pm 5.41^{\mathrm{a}}$ & $1.92 \pm 0.15^{\mathrm{a}}$ & $177.85 \pm 13.72^{\mathrm{a}}$ & $3.72 \pm 0.35^{\mathrm{a}}$ \\
Control & $4.54 \pm 0.33$ & $0.95 \pm 0.06$ & $71.76 \pm 6.62$ & $2.34 \pm 0.21$
\end{tabular}

Comparison with the normal control group, ${ }^{\mathrm{a}} \mathrm{P}<0.01$. CRP, C-reactive protein; IL-10, interleukin-10; PLA2, phospholipase A2; Fbg, fibrinogen $\beta$ polypeptide chain gene.

Treatment methods. After confirmed diagnosis, the study group underwent control of original trauma, early anti-shock treatment, protection of major organs, and support of gastrointestinal and parenteral nutrition. Acid-base imbalance and electrolyte disturbances were corrected and antibiotics were applied to prevent infection of patients. The study group was then treated with emergency surgery or damage control technologies.

Testing methods. Level changes of CRP, IL-10, PLA2 and Fbg in serum prior to surgery and $24 \mathrm{~h}$, and 5 and 7 days thereafter were closely monitored. IV fluids $(4 \mathrm{ml})$ were collected at the abovementioned time-points, and applied for hypothermal differential centrifugation. Serum was kept for testing. ELISA was used to detect the CRP level [detection kits provided by Xinran Biological Technology Co., Ltd. (Shanghai, China)]; IL-10 concentration [detection kits provided by Shanghai Wanjiang Biotech Co. (Shanghai, China)]; PLA2 level [detection kits provided by Shanghai Jining Biological Research Co. (Shanghai, China)]; and the Fbg level [detection kits of Fbg were provided by Beijing Bai'aoLaiBo Technology Co. (Beijing, China)]. All the detection kits were used according to the manufacturer's instructions.

Receiver operating characteristic (ROC) curve. ROC curve was used to detect the accuracy of Fbg in the prognosis of patients with traumatic fracture of lower limb. The area under the ROC curve was used as a summary index for the ROC curve. Ther ROC graph was generated using SPSS 20.0 statistical software (SPSS, Inc., Chicago, IL, USA).

Statistical analysis. SPSS 20.0 statistical software was used for statistical analysis. The t-test and Pearson linear correlation analysis were carried out. $\mathrm{P}<0.05$ was considered to indicate a statistically significant difference.

\section{Results}

Comparison of CRP, IL-1, PLA2 and Fbg of the two groups. Preoperative levels of CRP, IL-1, PLA2 and Fbg were markedly higher than those of the normal control group $(\mathrm{P}<0.01)$ (Table I).

Dynamic changes of CRP at different time-points. Ninety lower extremity trauma patients were treated successfully via surgery and their conditions were effectively controlled.

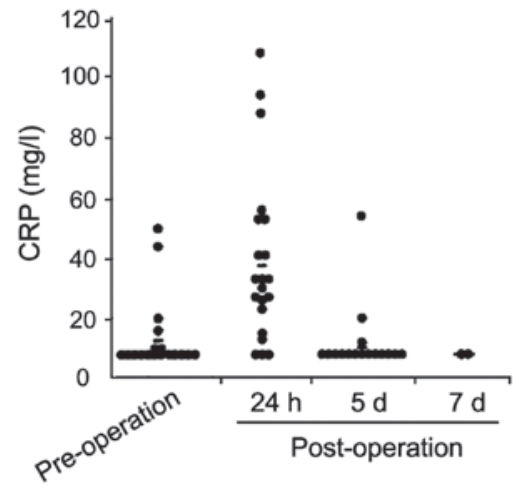

Figure 1. Dynamic changes of CRP at different time-points. CRP, C-reactive protein.

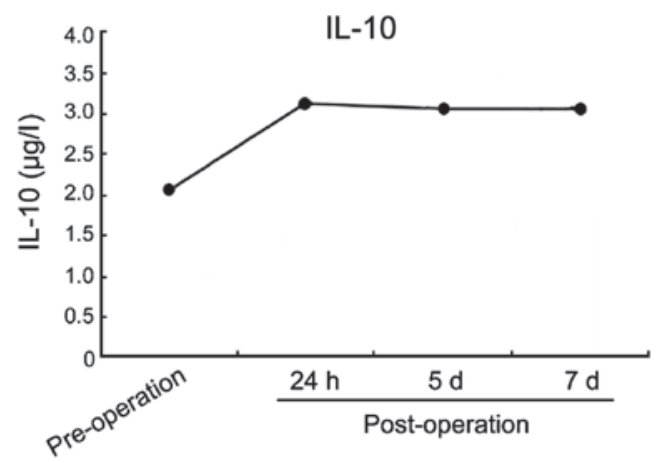

Figure 2. Dynamic changes of IL-10 at different time-points (x-axis, time-points of detection). IL-10, interleukin-10.

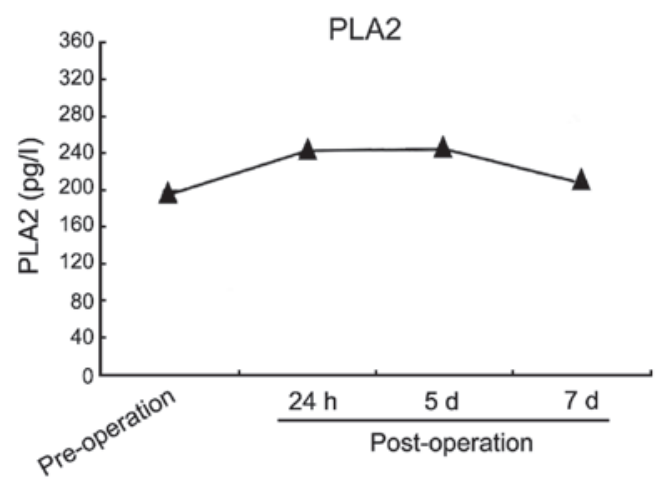

Figure 3. Dynamic changes of PLA2 at different time-points (x-axis, time-points of detection). PLA2, phospholipase A2.

Trauma patients were found with emergent CRP synthesis $24 \mathrm{~h}$ after surgery and the CRP concentration gradually decreased with the control and healing of trauma (Fig. 1).

Dynamic changes of IL-10 at different time-points. IL-10 level of trauma patients was increased during the first $24 \mathrm{~h}$ after operation and then gradually decreased (Fig. 2).

Dynamic changes of PLA2 at different time-points. PLA2 level of trauma patients was increased before operation and during the first $24 \mathrm{~h}$ after operation, and then gradually decreased (Fig. 3). 


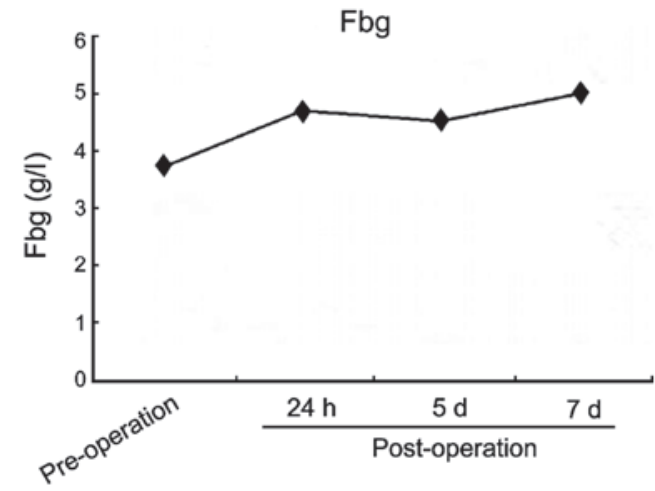

Figure 4. Dynamic changes of Fbg at different time-points. Fbg, fibrinogen $\beta$ polypeptide chain gene.

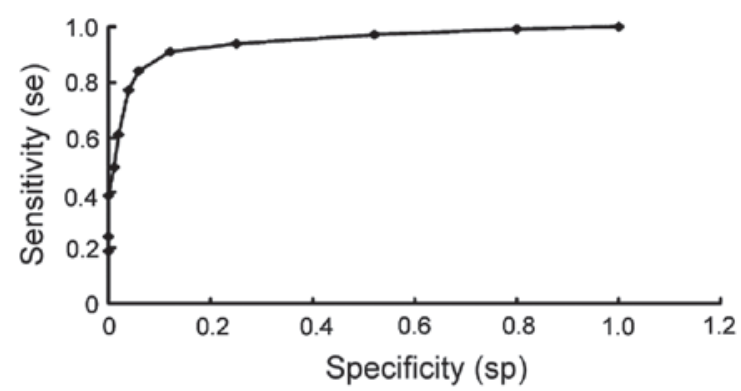

Figure 5. ROC graph of Fbg concentration (y-axis, sensitivity; $x$-axis, specificity). Fbg, fibrinogen $\beta$ polypeptide chain gene.

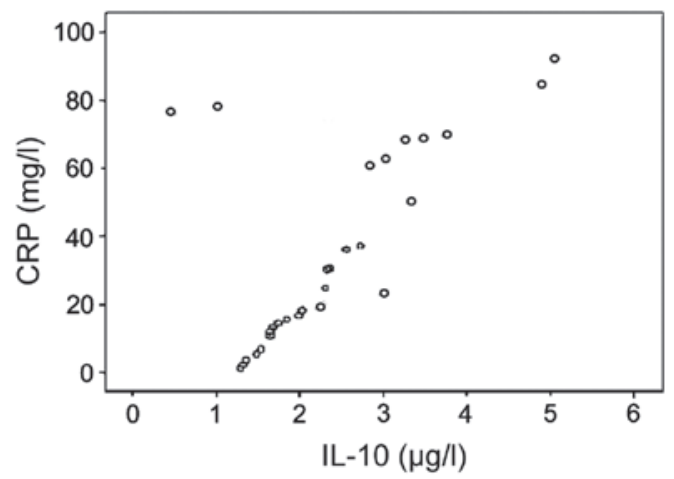

Figure 6. Correlation between levels of CRP and IL-10 of trauma patients. CRP, C-reactive protein; IL-10, interleukin-10.

Dynamic changes of Fbg at different time-points. The Fbg level of trauma patients was increased before operation and during the first $24 \mathrm{~h}$ after operation. The Fbg level gradually decreased and finally gradually increased again (Fig. 4). The ROC curve showed that the forming sensitivity and specificity of DVT was determined by separate observation of Fbg when Fbg $>4.71 \mathrm{~g} / \mathrm{l}$ was defined as 100 and $22.58 \%$, respectively (Fig. 5).

Corrective analysis of some indicators. CRP levels in serum of trauma patients were positively correlated with the IL-10 level $(r=0.634, \mathrm{P}<0.05)$ (Fig. 6). PLA2 levels in the serum of trauma patients were positively correlated with that of the IL-10 level $(\mathrm{r}=0.617, \mathrm{P}<0.05)$ (Fig. 7).

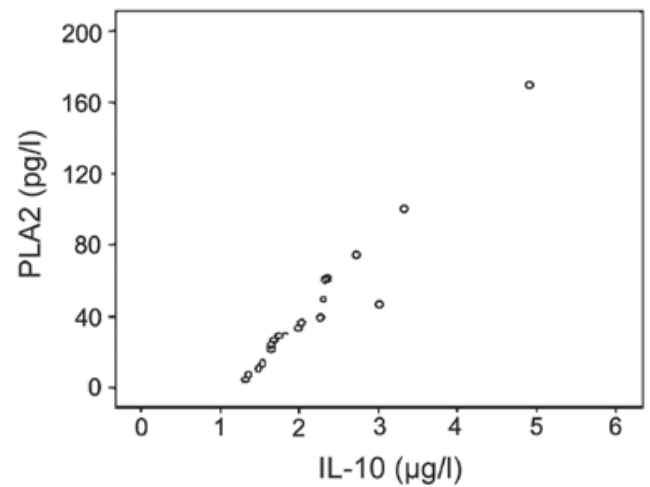

Figure 7. Correlation between the levels of PLA2 and IL-10 of trauma patients. PLA2, phospholipase A2; IL-10, interleukin-10.

\section{Discussion}

CRP is a sensitive and non-specific inflammatory marker of infection or traumas of human beings (10-12). Previous findings have shown that the CRP concentration in patients suffering bacterial infection is closely related to their trauma progression (13-15). If patients are successfully treated or their status improves, their CRP level is also reduced to normal level from high concentration. The present study has traced the level change of CRP in patients with lower extremity trauma and found that the peak level of CRP is evident $24 \mathrm{~h}$ after the operation as a result of emergency reaction. The CRP level was then decreased as the trauma became controlled and healed. However, if the patient CRP level continued to increase after operation, patients suffered complicated infection (16), and were given high attention. Patients with lower extremity trauma in the present study were successfully treated via surgery and no case of infection was observed. Therefore, an increase of CRP level was not observed after operation.

IL-10 is a newly found cytokine produced by Th2 cells. Previous studies have identified that IL-10 is closely related to infection after trauma (17-19). When the IL-10 level of trauma patients was increased, the incidence of complication and mortality also increased. However, few studies are available regarding the early change of level of IL-10 in serum of patients with lower extremity trauma (19). Our data showed that the level of IL-10 prior to surgery and during the $24 \mathrm{~h}$ after operation was increased and then decreased, suggesting that patients with lower extremity trauma were treated with relatively successful operations and their postoperative traumas were effectively controlled. Of note, IL-10 can be used for the prediction of progression of lower extremity trauma.

PLA2, an important enzyme, can release lipid mediator that actively reacts with inflammation, pathological changes of tissue damage and becomes involved in intra- and extracellular metabolic regulation (20). Previously, it was shown that, compared with healthy individuals, patients with acute pancreatitis and multiple organ failure have a higher concentration of PLA2 in their serum $(21,22)$. The present study revealed that the PLA2 level of trauma patients was increased prior to surgery and during the first $24 \mathrm{~h}$ after operation, and then gradually decreased. Thus, PLA2 is an important indicator in determining the injury degree of lower extremity trauma. 
Fgb has drawn increasing attention for its application in determining thrombosis $(3,23)$. Following lower extremity fractures and operations, lower limbs experienced a higher incidence of DVT as a result of vein injuries of different degrees. Clinical manifestations of DVT are not typical at early stages. Therefore, it is difficult to achieve accurate diagnosis and false-positive and -negative conditions become evident $(24,25)$. Therefore, early observation and diagnosis of DVT has become a study hotspot and challenge. The present study showed that the Fbg level of trauma patients was increased prior to surgery and during the first $24 \mathrm{~h}$ after operation, and then gradually decreased before gradually increasing again, suggesting that the incidence of DVT required attention when the Fbg level gradually increased following surgery. In the present study, the forming sensitivity and specificity of DVT was determined by separate observation of Fbg when Fbg $>4.71 \mathrm{~g} / 1$ was defined as 100 and $22.58 \%$, respectively. Notably, Fgb concentration increased in the serum of patients with lower extremity trauma and the change of Fgb index can be used to determine and prevent DVT formation.

A correlative analysis on some indicators found that CRP levels in the serum of trauma patients were positively correlated with the IL-10 level $(\mathrm{r}=0.634, \mathrm{P}<0.05)$. Additionally, PLA2 levels in the serum of trauma patients were positively correlated with the IL-10 level $(\mathrm{r}=0.617, \mathrm{P}<0.05)$. Therefore, levels of CRP and PLA2 increase as the level of IL-10 increases in patients with lower extremity trauma. Furthermore, levels of CRP and PLA2 decreased as the level of IL-10 decreased in patients with lower extremity trauma.

In conclusion, detection of the dynamic changes of levels of CRP, IL-10, PLA2 and Fbg in serum during the clinical diagnosis and treatment of lower extremity trauma patients may be useful regarding progression and provide valuable theoretical clues and a basis for prediction and determining their treatment effect and prognosis.

\section{References}

1. Azarpira N, Nikeghbalian S, Kazemi K, Geramizadeh B, Malekpour Z and Malek-Hosseini SA: Association of increased plasma interleukin-6 and TNF- $\alpha$ levels in donors with the complication rates in liver transplant recipients. Int J Organ Transplant Med 4: 9-14, 2013.

2. Mahar P: Lower extremity trauma. In: Berman's Pediatric Decision Making. 5th Edition. pp442-447, 2011.

3. Mok JM, Pekmezci M, Piper SL, Boyd E, Berven SH, Burch S, Deviren V, Tay B and Hu SS: Use of C-reactive protein after spinal surgery: comparison with erythrocyte sedimentation rate as predictor of early postoperative infectious complications. Spine 33: 415-421, 2008.

4. Harris AM, Althausen PL, Kellam J, Bosse MJ and Castillo $\mathrm{R}$ : Complications following limb-threatening lower extremity trauma. J Orthop Trauma 23: 1-6, 2009.

5. Gando S: Tissue factor in trauma and organ dysfunction. Semin Thromb Hemost 32: 48-53, 2006.
6. Gando S, Nanzaki S, Morimoto Y, Ishitani T and Kemmotsu O: Tissue factor pathway inhibitor response does not correlate with tissue factor-induced disseminated intravascular coagulation and multiple organ dysfunction syndrome in trauma patients. Crit Care Med 29: 262-266, 2001.

7. Waydhas C, Nast-Kolb D, Trupka A,Zettl R, Kick M, Wiesholler J, Schweiberer L and Jochum M: Posttraumatic inflammatory response, secondary operations, and late multiple organ failure. J Trauma 40: 624-630, 1996.

8. Giannoudis PV, Smith RM, Perry SL, Windsor AJ, Dickson RA and Bellamy MC: Immediate IL-10 expression following major orthopaedic trauma: relationship to anti-inflammatory response and subsequent development of sepsis. Intensive Care Med 26: 1076-1081, 2000

9. Lindström T, Gullichsen E, Heinonen O, Grönroos J, Nevalainen $\mathrm{T}$ and Niinikoski J: Group II phospholipase A2 in serum after knee surgery and intramedullary nailing of tibial shaft fracture. Injury 28: 169-171, 1997.

10. Holtslag HR, van Beeck EF, Lindeman E and Leenen LP: Determinants of long-term functional consequences after major trauma. J Trauma 62: 919-927, 2007.

11. Simmel S and Bühren V: Surviving multiple trauma - what comes next? The rehabilitation of seriously injured patients. Unfallchirurg 112: 965-974, 2009 (In German).

12. Manly DA, Boles J and Mackman N: Role of tissue factor in venous thrombosis. Annu Rev Physiol 73: 515-525, 2011.

13. Kim JW, Oh CW, Oh JK, Baek SG, Lee BJ, Hong HP and Min WK: The incidence and the risk factors of venous thromboembolism in Korean patients with pelvic or acetabular fractures. J Orthop Sci 19: 471-477, 2014.

14. Yi X, Zhu J, Wei M, Li J, Chen J, Wang Y, Shen E and Hu B: Risk factors of venous thrombosis in patients with ankle fractures. Int Angiol 33: 324-328, 2014.

15. Mulsow J, O'Toole GC and McManus F: Traumatic lower limb fractures following complete spinal cord injury. Ir Med J 98: 141-142, 2005.

16. Peivandi MT, Yusof-Sani MR and Amel-Farzad H: Exploring the reasons for orthopedic implant failure in traumatic fractures of the lower limb. Arch Iran Med 16: 478-482, 2013.

17. Cross AM, Davis C, Penn-Barwell J, Taylor DM, De Mello WF and Matthews JJ: The incidence of pelvic fractures with traumatic lower limb amputation in modern warfare due to improvised explosive devices. J R Nav Med Serv 100: 152-156, 2014.

18. Besemann M: Physical rehabilitation following polytrauma. The canadian forces physical rehabilitation program 2008-2011. Can J Surg 54: S135-S141, 2011.

19. Rowley AH and Shulman ST: Recent advances in the understanding and management of kawasaki disease. Curr Infect Dis Rep 12: 96-102, 2010.

20. Rowley AH and Shulman ST: New developments in the search for the etiologic agent of Kawasaki disease. Curr Opin Pediatr 19: 71-74, 2007.

21. Harris KC and Hosking MC: Persistent fever in an infant: incomplete Kawasaki disease. CMAJ 183: 2009-2013, 2011.

22. Beken B, Unal S, Cetin M and Gümrük F: The relationship between hematological findings and coronary artery aneurysm in kawasaki disease. Turk J Haematol 31: 199-200, 2014.

23. Schalek P, Hornáčková Z and Hahn A: The relationship of $\mathrm{C}$-reactive protein levels and positive culture with quality of life in acute rhinosinusitis. Patient Prefer Adherence 9: 185-189, 2015.

24. Wallace GF: Rehabilitation after major extremity trauma. Clin Podiatr Med Surg 31: 585-595, 2014.

25. Inaba K, Siboni S, Resnick S, Zhu J, Wong MD, Haltmeier T, Benjamin E and Demetriades D: Tourniquet use for civilian extremity trauma. J Trauma Acute Care Surg 79: 232-237, 2015. 